\title{
Efficacy of Cardiac Blood Pool Activity in Hepatobiliary Scintigraphy for Diagnosis of Neonatal Hepatitis in Patients with Suspected Biliary Atresia
}

\author{
Noorjahan Khan, Shamim MF Begum, Pupree Mutsuddy and Sadia Sultana \\ National Institute of Nuclear Medicine and Allied Sciences (NINMAS), Dhaka \\ Correspondence Address: Dr. Noorjahan Khan, M. Phil (Nuclear Medicine), National Institute of Nuclear Medicine \& Allied \\ Sciences (NINMAS), Email: nupurpasha@yahoo.com
}

\begin{abstract}
Background: Biliary atresia (BA) is the commonest fatal liver disorder in children. It results from developmental anomaly due to fibrosis of extra hepatic bile ducts. The reported global incidence of biliary atresia varies from $5 / 100,000$ to $32 / 100,000$ live births. Accurate diagnosis is important before 8 weeks of age, since hepatoportoenterostomy (Kasai's procedure) has a $\mathbf{9 0 \%}$ success rate at this stage whereas success rate drops to $20 \%$ beyond 3 months. On the other hand, neonatal hepatitis (NH) which is other most common cause of neonatal cholestasis (NC) needs conservative treatment. So, differentiation of biliary atresia from neonatal hepatitis is very crucial. Hepatobiliary scintigraphy (HBSG) is most popular method to differentiate biliary atresia from neonatal hepatitis. But in severe neonatal hepatitis the diagnosis become inconclusive when excretion of radiotracer in bowel become absent along with prolonged cardiac activity. Prolonged cardiac blood pool activity in hepatobiliary scintigraphy is an indication of hepatocyte destruction in neonatal hepatitis. The aim of the study was to assess the efficacy of cardiac blood pool in hepatobiliary scintigraphy for diagnosis of neonatal hepatitis in suspected biliary atresia patient.
\end{abstract}

Patients and methods: A total of 24 infants with history of conjugated hyperbilirubinaemia underwent HBSG showed prompt cardiac blood pool activity (9 infants) and also with prolonged cardiac blood pool (15 infants) with no excretion of radiotracer in bowel either in early or delayed images was included in the study. The findings of HBSG were compared with percutaneous liver biopsy findings and analyzed.

Result: Among 24 infants prolonged cardiac blood pool activity in HBSG was observed in $11(73.3 \%)$ BA infants and 4 (44.4\%) of $\mathrm{NH}$ infants. Absent bowel activity with prompt clearance of cardiac blood pool activity was observed in 4 (26.7\%) BA infants and 5 (55.6\%) NH infants. The difference was not statistically significant $(p>0.05)$. The calculated sensitivity of cardiac blood pool activity in HBSG for evaluation of $\mathrm{NH}$ was $44.4 \%$, specificity was $26.7 \%$, accuracy was $33.3 \%$, positive predictive value (PPV) was $26.7 \%$ and negative predictive value (NPV) was $44.4 \%$. The sensitivity, specificity, PPV, NPV and accuracy were low due to large number of false positive infants which might be due to complete obstruction of bile duct or due to early hepatocyte destruction (within 3 months of age).

Conclusion: Although prolonged cardiac blood pool activity was considered as diagnostic criteria for the diagnosis of $\mathrm{NH}$ in suspected BA patient but it could not be able to differentiate these two conditions.

Key words: Biliary aresia, Neonatal hepatitis, Hepatobiliary scintigraphy, Cardiac blood pool.

\section{INTRODUCTION}

Neonatal Cholestasis (NC) is defined as conjugated hyperbilirubinaemia occurs in the infant as a consequence of diminished bile flow. It accounts almost $30 \%$ of hepatobiliary disease of children. The usual presentations of $\mathrm{NC}$ are with variable degree of jaundice, dark urine, pale stool and enlarged liver. The two main causes of $\mathrm{NC}$ are biliary atresia (BA) and neonatal hepatitis $(\mathrm{NH})(1,2)$.

Jaundice in first 2 weeks after birth accounts in 2.4\% to $15 \%$ of newborns. It may be due to conjugated hyperbilirubinaemia or unconjugated hyperbilirubinaemia (3). Jaundice in infants is mostly unnconjugated bilirubin variety which resolves spontaneously without intervention. However, if jaundice persists beyond 2 weeks of age, $\mathrm{NC}$ or conjugated hyperbilirubinemia must be considered in 
the differential diagnosis. Conjugated hyperbilirubinemia is never physiologic and if untreated metabolic dysfunction and serious hepatobiliary consequence like chronic liver disease may occur. Hence identifying the underlying cause of conjugated hyperbilirubinemia or NC is essential to ensure appropriate treatment in time for optimal prognosis. Even though in some cases the specific treatment may not be available, early medical management and optimization of nutrition of the infants with cholestasis can prevent complications (4). $\mathrm{BA}$ is the end result of a destructive, inflammatory process that affects intra and extra hepatic bile ducts, leading to fibrosis and obliteration of the biliary tract with the development of biliary cirrhosis (1). Infant presented with $\mathrm{NC}$ due to $\mathrm{BA}$ requires surgical intervention. $\mathrm{NH}$ is the inflammation of liver occurs in early infancy usually between one and two months after birth and the reported incidence of $\mathrm{NH}$ is 1 in 8000 live births and needs adequate medical management $(5,6)$. Hence, differentiation between BA and $\mathrm{NH}$ is very important for treatment planning and to avoid unnecessary intervention.

No single test can distinguish BA form $\mathrm{NH}$, clinically it is difficult and biochemical tests are inconclusive. Various imaging modalities are currently used for the diagnosis of BA and $\mathrm{NH}$. These are abdominal ultrasonography, radionuclide hepatobiliary scintigraphy, computed tomography, magnetic resonance imaging and percutaneous trans-hepatic cholangiography. Some of these modalities are invasive while others do not provide functional information (7).

HBSG is a non-invasive, popular nuclear medicine imaging modality. It is widely incorporated in an early investigation work up of NC. It plays an important role in the diagnosis of BA from other causes of cholestasis in early stage of the disease (8). 99m Technetium ( ${ }^{99 m}$ Tc) labeled iminodiacetic acid (IDA) is the most commonly used radiopharmaceutical for HBSG. Normally HBSG is characterized by rapid uptake of ${ }^{99 \mathrm{~m}} \mathrm{Tc}$ IDA by the hepatocyte, rapid clearance of cardiac blood-pool activity followed by sequentially visualization of activity in intrahepatic and extra hepatic biliary ducts, gallbladder (GB) and upper intestine. Usually peak hepatic uptake occurs within 5 minutes, cardiac blood pool activity clears within 15 minutes, the GB may be visualized within 10 minutes. Occasionally GB may not be visualized in neonates, which is physiological. The intestinal or bowel activity is usually seen between 20 to 30 minutes (9).

In $\mathrm{NC}$ visualization of tracer activity into the bowel excludes BA. According to Moyer et al. the sensitivity of hepatobiliary scintigraphy in diagnosis of BA is as high as $83-100 \%$ (10). In infants with BA within three months of age; hepatocyte functions remain normal and hepatobiliary scinigraphy shows prompt or normal hepatic uptake with rapid cardiac blood pool activity clearance. Presence of activity in the bowel is consistent with $\mathrm{NH}$ or other causes of NC. However bowel activity may be absent in case of severe NH or in hepatocyte destruction. Hepatic dysfunction in $\mathrm{NH}$ causes delayed clearance of tracer from the blood pool leads to prolonged cardiac blood pool activity (11).

It is well accepted that no single clinical, biochemical or imaging study is entirely satisfactory in the evaluation of NC. Final diagnosis can be made by correlation of data from clinical history, biochemical values, imaging and liver biopsy (12).

\section{PATIENTS AND METHODS}

This prospective observational type of study was carried out in National Institute of Nuclear Medicine \& Allied Sciences (NINMAS), BSMMU campus, Dhaka from July, 2015 to December, 2016. Infants who were suspected for biliary atresia due to absent 
bowel activity in HBSG were included in this study. Infants who were more than 3 months of age, with history of unconjugated hyperbilirubinaemia and also with co-morbid disease such as renal failure, bleeding disorder, congenital anomaly were excluded.

All the infant's guardians were informed about the potential risk and benefit of the procedure and informed written consent was taken from each patient's guardian before the procedure. Details history and patient's biochemical tests findings were recorded from their medical file. HBSG was performed with ${ }^{99 \mathrm{~m}} \mathrm{Tc}$ BrIDA or Mebrofenin which was given intravenously according to body weight. The patient was scanned under dual headed gamma camera with high resolution collimator using $256 \mathrm{X}$ 256 matrix in supine position. Then sequential images were obtained at $5,10,15,20,25,30,45$ and 60 minutes in anterior view. Additional images in right lateral position were taken when required. Delayed images were taken at 2, 4 and 24 hours. When there was prompt hepatic uptake and prompt blood pool clearance $(<15 \mathrm{~min})$ but no excretion of radiotracer in the bowel those were reported as suspected BA. The prolonged cardiac blood pool activity ( $>15 \mathrm{~min}$ ) and no visualization tracer in the bowel were considered as inconclusive. Liver biopsy was done in 24 cases. If section showed core of liver tissue, portal tract showed mild fibrosis and infiltration of chronic inflammatory cells; the diagnosis of BA was confirmed. If section area showed moderate number of chronic inflammatory cells, no bile duct proliferation; the diagnosis was hepatitis.

The collected data were compiled and analyzed using computer based software MS Excel and SPSS (Statistical Package for Social Science) version 20 for windows by appropriate statistical methods. In each analysis level of significance was 0.05 and $p$ value $<$ 0.05 was considered significant. The sensitivity, specificity, PPV, NPV and accuracy of cardiac blood pool of HBSG were measured.

\section{RESULTS}

Among 24 infants (15 males, 9 females) liver biopsy confirmed BA in 15 infants and $\mathrm{NH}$ in 9 infants. BA group is mentioned as group $\mathrm{A}$ and $\mathrm{NH}$ group is mentioned as group B.

It was observed that $5(33.3 \%)$ infants belonged to $<2$ months of age in group A and 1 (11.1\%) infant in group B. A total $10(66.7 \%)$ infants belong to 2-3 months of age in group A and 8 (89.9\%) in group B. The mean age was $2.2 \pm 0.7$ months in group $\mathrm{A}$ and $2.3 \pm 0.6$ months in group B. The difference was not statistically significant $(p>0.05)$ between two groups (Table 1).

Table 1: Distribution of the study population by age $(n=24)$

\begin{tabular}{|c|c|c|c|c|c|}
\hline \multirow[t]{2}{*}{ Age (in month) } & \multicolumn{2}{|c|}{ Group A (n=15) } & \multicolumn{2}{|c|}{ Group B $(\mathrm{n}=9)$} & \multirow[t]{2}{*}{$p$ value } \\
\hline & $\mathrm{n}$ & $\%$ & $\mathrm{n}$ & $\%$ & \\
\hline$<2$ & 5 & 33.3 & 1 & 11.1 & \\
\hline $2-3$ & 10 & 66.7 & 8 & 89.9 & \\
\hline \multirow[t]{2}{*}{$\operatorname{Mean} \pm \mathrm{SD}$} & \multirow{2}{*}{\multicolumn{2}{|c|}{$2.2 \pm 0.7$}} & \multicolumn{2}{|c|}{$2.3 \pm 0.6$} & \\
\hline & & & & & 0.724 \\
\hline Range (min, $\max )$ & \multicolumn{2}{|c|}{1,3} & \multicolumn{2}{|c|}{$1.5,3$} & \\
\hline
\end{tabular}

Male to female ratio was $1.14: 1$ in group $\mathrm{A}$ and in group B the ratio was 3.5:1.

In the study, $11(73.3 \%)$ infants of group A had prolonged cardiac blood pool activity ( $>15$ minutes) and $4(44.4 \%)$ infants in group B had prolonged cardiac blood pool. Prompt clearance of cardiac blood pool activity $(<15$ minutes) were in $4(26.7 \%)$ infants of group A and 5 (55.6\%) infants in group B (Table 2).

Table 2: Distribution of the study population by cardiac blood pool activity in Hepatobiliary Scintigraphy $(n=24)$

\begin{tabular}{lccccc}
\hline $\begin{array}{l}\text { Cardiac blood pool activity } \\
\text { (in min) }\end{array}$ & Group A (n=15) & Group B (n=9) & $p$ value \\
\hline & $\mathrm{n}$ & $\%$ & $\mathbf{n}$ & $\%$ & \\
$>15$ min (prolonged) & 11 & 73.3 & 4 & 44.4 & \\
$\leq 15$ min (prompt) & 4 & 26.7 & 5 & 55.6 & 0.159 \\
\hline
\end{tabular}


Among the 24 infants, in 15 infants with prolonged blood pool activity, liver biopsy confirmed $\mathrm{NH}$ in 4 infants (true positive) and BA in 11 infants (false positive). Whereas, in 9 infants with prompt blood pool activity liver biopsy showed $\mathrm{NH}$ in 5 infants (false negative) and BA in 5 (true negative) (Table 3).

Table 3: Association between cardiac blood pool activity in hepatobiliary scintigraphy with liver biopsy $(n=24)$

\begin{tabular}{lccc}
\hline $\begin{array}{l}\text { Cardiac blood pool } \\
\text { activity (min) }\end{array}$ & \multicolumn{2}{c}{ Liver biopsy } & Total \\
\cline { 2 - 3 } & NH & BA & \\
\hline$>\mathbf{1 5}$ (prolonged) & 4 & 11 & 15 \\
$<\mathbf{1 5}$ (prompt) & 5 & 4 & 9 \\
\hline Total & 9 & 15 & 24 \\
\hline
\end{tabular}

The sensitivity, specificity, PPV, NPV of cardiac blood pool activity in HBSG for diagnosis of $\mathrm{NH}$ were $44.4 \%$, $26.7 \%, 26.7 \%$ and $44.4 \%$ respectively. The overall accuracy was $33.3 \%$.

\section{DISCUSSION}

24 infants, below 3 months of age with history of jaundice for more than 14 days were enrolled in this study. In this present study, 5 (33.3\%) infants less than 2 months of age and $10(66.7 \%)$ infants between 2-3 months age were in group A. In group B, $1(11.1 \%)$ infant was less than 2 months and $8(88.9 \%)$ were between 2-3 months of age. The mean age \pm SD of group A infants was $2.2 \pm 0.7$ months and in group B was $2.3 \pm$ 0.6 months. The mean age difference was statistically not significant ( $p>0.05$ ) between the two groups of infants (Table 1). Study conducted by Gilmour et al. found the age range of the NC infants 2 days to 4 months, with a mean age of about 2 months 1 week, supporting this study (13). In a study by Ahmed et al. the mean age \pm SD was $4.87 \pm 2.46$ months in infants of $\mathrm{NC}$, which was higher than this study, which might be due the inclusion of more than 3 months old infants (14).

This study showed male to female ratio $1.14: 1$ in group $A$ and 3.5:1 in group B, indicating male predominance in both groups. Male predominance was also reported by
Gilmour et al. with a male female ratio of 2.07:1 (13). Similar observation was also reported by Kelly \& Stanton and Howman-Giles et al. $(3,9)$.

Prolonged cardiac blood pool activity is an indicator of hepatic dysfunction due to $\mathrm{NH}$ (15). However prolonged cardiac blood pool activity was evident not only in $\mathrm{NH}$ but also in BA infants. Hepatobiliary scan with absent bowel activity and prolonged cardiac blood pool activity was observed in 11 (73.3\%) infants of group A and 4 (44.4\%) infants of B. Absent bowel activity with prompt clearance of cardiac blood pool activity was observed in 4 (26.7\%) infants of group A and 5 (55.6\%) infants of group B. This study showed increased frequency of prolonged cardiac blood pool activity in BA comparing to $\mathrm{NH}$, but the difference was not statistically significant ( $p>0.05$ ) (Table 2). Prolonged cardiac blood pool activity in BA infants is in disagreement, whereas the infants with prolonged cardiac blood pool in group B is in agreement.

The association between cardiac blood pool activity with liver biopsy was analyzed (Table 3). Among 24 infants prolonged cardiac blood pool activity was observed in 15 infants, out of them only 4 infants were positive for $\mathrm{NH}$ and 11 infants were positive for BA. Rest 9 infants showed prompt cardiac blood pool activity, out of them 5 infants were positive for $\mathrm{NH}$ and 4 infants were positive for BA. In infants when HBSG showed prolonged cardiac blood pool activity and liver biopsy confirmed NH these cases were TP. The infants showed prolonged cardiac blood pool activity in HBSG and liver biopsy showed BA, those cases were false positive FP. Infants showed prompt cardiac blood pool activity HBSG and liver biopsy confirmed NH these cases were FN. When HBSG showed prompt cardiac blood pool activity and liver biopsy confirmed BA those cases were true negative TN. The calculated sensitivity of cardiac blood pool activity in HBSG for diagnosis of $\mathrm{NH}$ was $44.4 \%$, specificity was $26.7 \%$, accuracy was $33.3 \%$, PPV was $26.7 \%$ and NPV was $44.4 \%$. Low sensitivity, 
specificity, PPV, NPV and accuracy of blood pool activity in diagnosis $\mathrm{NH}$ indicates that it cannot be used as a reliable tool in HBSG in patients with NC. Prolonged blood pool activity in BA might be due to complete obstruction of bile duct or due to early hepatocyte destruction (within 3 months of age). Though prolonged blood pool activity is considered as a predictor for diagnosis of $\mathrm{NH}$, no such study was found utilizing blood pool activity in differentiating $\mathrm{NH}$ from BA for comparison.

\section{CONCLUSION}

Accurate diagnosis of underlying causes of $\mathrm{NC}$ is challenging. During HBSG absent radiotracer excretion in bowel is consistent with BA. Similar findings in neonatal hepatitis with associated hepatocyte destruction makes the study inconclusive for diagnosis of BA. Prolonged cardiac blood pool activity in HBSG is an indication of hepatocyte dysfunction in NH. Although prolonged cardiac blood pool activity was considered as diagnostic criteria for diagnosis of $\mathrm{NH}$. But prolonged cardiac blood pool was also evident in BA infant. In this study, the prolonged cardiac blood pool activity couldn't be able to diagnosis $\mathrm{NH}$ in patient with suspected BA within 3 months of age infants. Other modalities like intraoperative cholangiography, MRCP, follow-up biopsy are required to reach the final diagnosis. In future, further exploration of the study can be done with larger sample size.

\section{REFERENCES}

1. Balistreri WF, Grand R, Hoofnagle JH, Suchy FJ, Ryckman FC and Perlmutter DH et al. Biliary atresia: current concepts and research directions. Summary of a symposium. Hepatology 1996;23(6):1682-92.

2. Bhatia V, Bavdekar A, Matthai J, Waikar Y and Sibal A. Management of neonatal cholestasis: consensus statement of the Pediatric Gastroenterology Chapter of Indian Academy of Pediatrics. Indian Pediatr 2014;51(3):203-10.

3. Kelly DA and Stanton A, Jaundice in babies: implications for community screening for biliary atresia. BMJ 1995;310(6988):1172-1173.
4. Serinet MO, Wildhaber BE, Broue P, Lachaux A, Sarles J and Jacquemin $E$ et al. Impact of age at Kasai operation on its results in late childhood and adolescence: a rational basis for biliary atresia screening. Pediatrics 2009;123:1280-86

5. Krishnamurthy GT and Krishnamurthy S. Imaging and Quantification of Hepatobiliary Function. In Nuclear Hepatology, Springer Berlin Heidelberg, 2000: 93-127.

6. Dehghani SM, Haghighat M, Imanieh $\mathrm{MH}$ and Geramizasleh B. Comparison of different diagnostic methods in infants with cholestasis. World J Gastroenterol 2006;12(36):5893-6.

7. Miftari R, Alimanovic-Alagic R, Bicaj X, Shala Z, Nura A and Gerqari I et al. The Role of Hepato-biliary Scintigraphy in Differentiation of Bilary Atresia from Other Causes of Neonatal Jaundice. Acta Informatica Medica 2011;19(1):58.

8. Hownan-Giles R, Uren R, Berhard E and Dorney $\mathrm{S}$. Hepatobiliary Scintigraphy in infancy. J Nucl Med 1998;39(2):311-31

9. Hownan-Giles R, Moase A, Gaskin and Uren R. Hepatobiliary Scintigraphy in a Pediatric population: Determination of Hepatic Extraction Fraction by Deconvolution Analysis. J Nucl Med 1993;34:214-21.

10. Moyer V, Freese DK, Whitington PF, Olson AD, Brewer F and Colletti RB et al. Guideline for the evaluation of cholestatic jaundice in infants: recommendations of the North American Society for Pediatric Gastroenterology, Hepatology and Nutrition. J Pediatr Gastroenterol Nutr 2004;39(2):115-28.

11. Majd M, Reba RC and Altman RP. Effect of phenobarbital on 99m Tc-IDA scintigraphy in the evaluation of neonatal jaundice. Seminars in Nuclear Medicine 1981 July;11( 3):184-204.

12. Rouzrokh MOHSEN, Sobhiyeh MR and Heibatollahi M. The sensitivity, specificity, positive and negative predictive values of stool color test, triangular cord sign and hepatobiliaryscintigraphy in diagnosis of infantile biliary atresia. Iranian Red Crescent Medical Journal 2009;11(4):425.

13. Gilmour SM, Hershkop M, Reifen R, Gilday D and Roberts EA. Outcome of Hepatobiliary Scanning in Neonatal Hepatitis Syndrome. J Nucl Med 1997;38(8):1279-82.

14. Ahmed SR, Sultana S, Begum SMF, Alam F. Evaluation of Diagnostic Accuracy of Hepatobiliary Scintigraphy in Diagnosing Biliary Atresia at Institute of Nuclear Medicine and Ultrasound. Bangladesh J Nucl Med 2013;16(1):23-8.

15. Mettler Jr FA and Guiberteau MJ. Gastrointestinal Tract. In: Essentials of nuclear medicine imaging. 6th Ed. Philadelphia, PA 19103-2899, 2012:237-270. 